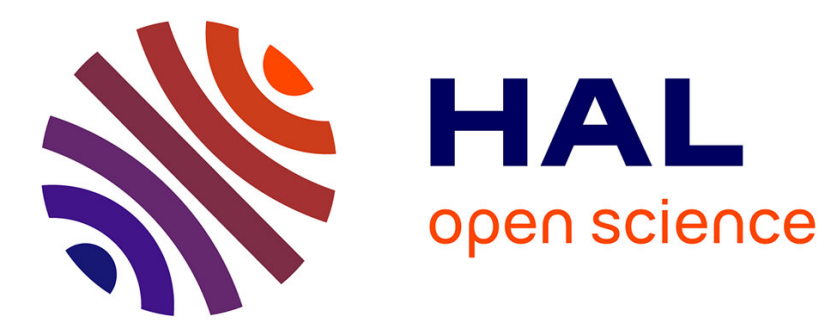

\title{
The Green-Kubo formula and the Onsager reciprocity relations in quantum statistical mechanics
}

\author{
Vojkan Jaksic, Yoshiko Ogata, Claude-Alain Pillet
}

\section{To cite this version:}

Vojkan Jaksic, Yoshiko Ogata, Claude-Alain Pillet. The Green-Kubo formula and the Onsager reciprocity relations in quantum statistical mechanics. Communications in Mathematical Physics, 2006, 265 (3), pp.721-738. 10.1007/s00220-006-0004-6 . hal-00005386

\section{HAL Id: hal-00005386 https://hal.science/hal-00005386}

Submitted on 15 Jun 2005

HAL is a multi-disciplinary open access archive for the deposit and dissemination of scientific research documents, whether they are published or not. The documents may come from teaching and research institutions in France or abroad, or from public or private research centers.
L'archive ouverte pluridisciplinaire HAL, est destinée au dépôt et à la diffusion de documents scientifiques de niveau recherche, publiés ou non, émanant des établissements d'enseignement et de recherche français ou étrangers, des laboratoires publics ou privés. 


\title{
The Green-Kubo formula and the Onsager reciprocity relations in quantum statistical mechanics
}

\author{
V. Jakšić ${ }^{1}$, Y. Ogata ${ }^{2,3}$, C.-A. Pillet ${ }^{2}$ \\ ${ }^{1}$ Department of Mathematics and Statistics \\ McGill University \\ 805 Sherbrooke Street West \\ Montreal, QC, H3A 2K6, Canada \\ ${ }^{2}$ CPT-CNRS, UMR 6207 \\ Université du Sud, Toulon-Var, B.P. 20132 \\ F-83957 La Garde Cedex, France \\ ${ }^{3}$ Department of Mathematical Sciences \\ University of Tokyo \\ Komaba,Tokyo,153-8914 Japan
}

June 15, 2005

Dedicated to David Ruelle on the occasion of his 70th birthday

\begin{abstract}
We study linear response theory in the general framework of algebraic quantum statistical mechanics and prove the Green-Kubo formula and the Onsager reciprocity relations for heat fluxes generated by temperature differentials. Our derivation is axiomatic and the key assumptions concern ergodic properties of non-equilibrium steady states.
\end{abstract}




\section{Introduction}

This is the first in a series of papers dealing with linear response theory in non-equilibrium quantum statistical mechanics. The three pillars of linear response theory are the Green-Kubo formula (GKF), the Onsager reciprocity relations (ORR), and the Central Limit Theorem. This paper and its sequels [JOP1, JOP2] deal with the first two. An introduction to linear response theory in the algebraic formalism of quantum statistical mechanics can be found in the recent lecture notes [AJPP1]. We emphasize that our program is concerned with purely thermodynamical (i.e. "non-mechanical") driving forces such as deviations of temperature and chemical potential from their equilibrium values.

The main result of this paper is an abstract derivation of the GKF and the ORR for heat fluxes. Various generalizations of our model and results (and in particular, the extension of GKF and ORR to heat and charge fluxes) are discussed in [JOP1]. Our abstract derivation directly applies to open quantum systems with free fermionic reservoirs previously studied in [Da, LeSp, BM, AM, JP2, FMU]. These applications are discussed in [JOP2, JOPP].

The mathematical theory of non-equilibrium quantum statistical mechanics has developed rapidly over the last several years. The key notions of non-equilibrium steady states (NESS) and entropy production have been introduced in [Ru1, Ru2, Ru3, JP1, JP2, JP3]. The general theory has been complemented with the development of concrete techniques for the study of non-equilibrium steady states [Ru1, JP2, FMU] and at the moment there are several classes of non-trivial models whose non-equilibrium thermodynamics is reasonably well-understood. The development of linear response theory is the natural next step in this program.

The GKF for mechanical perturbations has been studied in many places in the literature (see [BGKS, GVV1] for references and additional information). Mathematically rigorous results for thermodynamical perturbations are much more scarce. Our research has been partly motivated by the work of Lebowitz and Spohn [LeSp] who studied linear response theory for quantum Markovian semigroups describing dynamics of open quantum systems in the van Hove weak coupling limit. The ORR for directly coupled fermionic reservoirs have been discussed in [FMU] in first order of perturbation theory. The mean field theory aspects of ORR are discussed in [GVV2]. A fluctuation theorem related to linear response theory can be found in [TM]. Needless to say, physical aspects of linear response theory are discussed in many places in the literature, and in particular in the classical references [DGM, KTH]. An exposition in spirit close to our approach can be found in [Br, Zu, ZMR1, ZMR2]. Linear response theory in classical non-equilibrium statistical mechanics has been reviewed in [Ru4, RB].

Our model can be schematically described as follows. Consider two infinitely extended quantum systems which for convenience we will call the left, L, and the right, $\mathrm{R}$, system. The systems $\mathrm{L}$ and $\mathrm{R}$ may have additional structure (for example, in the case of open quantum systems L will consists of a "small" (finite level) system $\mathcal{S}$ coupled to several reservoirs and $\mathrm{R}$ will be another reservoir coupled to the small system, see Figure 1).

Assume that initially the system $\mathrm{L}$ is in thermal equilibrium at a fixed (reference or equilibrium) inverse temperature $\beta_{\mathrm{L}}=\beta$, and that the system $\mathrm{R}$ is in thermal equilibrium at inverse temperature $\beta_{\mathrm{R}}$. The thermodynamical force $X$ is equal to the deviation of the inverse temperature of the right system from the equilibrium value $\beta$,

$$
X=\beta-\beta_{\mathrm{R}} .
$$

Assume that the systems $\mathrm{L}$ and $\mathrm{R}$ are brought into contact. One expects that under normal conditions the joint system $\mathrm{L}+\mathrm{R}$ will rapidly settle into a steady state $\omega_{X,+}$. If $X=0$, then $\omega_{0,+} \equiv \omega_{\beta}$ is the joint thermal equilibrium state of $\mathrm{L}+\mathrm{R}$ characterized by the Kubo-Martin-Schwinger (KMS) condition. If $X \neq 0$, then $\omega_{X,+}$ is a non-equilibrium steady state (NESS) characterized by non-vanishing entropy production

$$
\operatorname{Ep}\left(\omega_{X,+}\right)=X \omega_{X,+}(\Phi)>0
$$

where $\Phi$ is the observable describing the heat flux out of R. For additional information about this setup we refer the reader to [Ru1, Ru2, Ru3, JP1, JP2, JP3].

The Green-Kubo linear response formula asserts that if the joint system is time-reversal invariant and the observable $A$ is odd under time-reversal, then

$$
\left.\partial_{X} \omega_{X,+}(A)\right|_{X=0}=\frac{1}{2} \int_{-\infty}^{\infty} \omega_{\beta}\left(A \Phi_{t}\right) \mathrm{d} t
$$




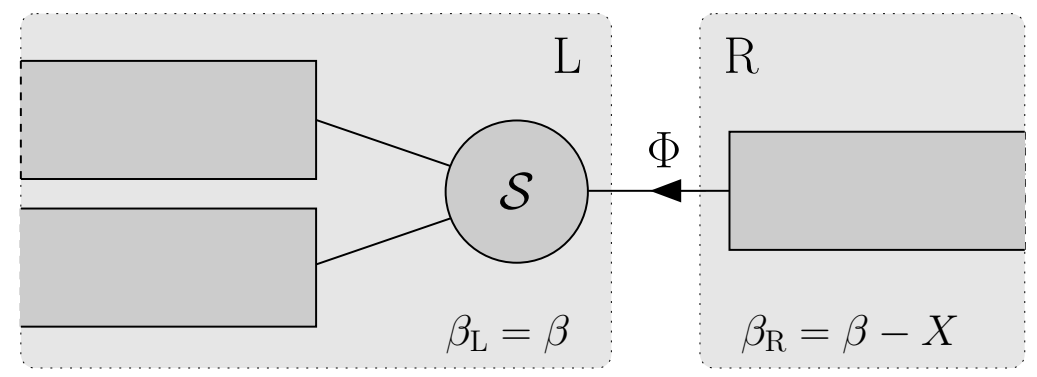

Figure 1: An open quantum system represented as $\mathrm{L}+\mathrm{R}$.

where $t \mapsto \Phi_{t}$ is the dynamics in the Heisenberg picture. This celebrated formula relates the linear response to the equilibrium correlations and is a mathematical expression of the fluctuation-dissipation mechanism in statistical mechanics.

The ORR are direct consequences of the GKF. Consider three systems A, B, C, which are respectively in thermal equilibrium at inverse temperatures $\beta-Y, \beta$, and $\beta-X$. Assume that the systems are brought into contact by interactions which couple A with B and B with $\mathrm{C}$. Let $\omega_{X, Y,+}$ be the non-equilibrium steady state of the joint system and $\Phi_{\mathrm{A}}, \Phi_{\mathrm{C}}$ the observable which describe the heat flow out of $\mathrm{A}, \mathrm{C}$ (see Figure 2). If the system is time-reversal invariant, then $\Phi_{\mathrm{A}}$ and $\Phi_{\mathrm{C}}$ are odd under time-reversal.

Assume that the functions $\omega_{X, Y,+}\left(\Phi_{\mathrm{C}}\right)$ and $\omega_{X, Y,+}\left(\Phi_{\mathrm{A}}\right)$ are differentiable at $X=Y=0$. The kinetic transport coefficients are defined by

$$
\begin{aligned}
& \left.L_{\mathrm{A}} \equiv \partial_{X} \omega_{X, Y,+}\left(\Phi_{\mathrm{A}}\right)\right|_{X=Y=0}, \\
& \left.L_{\mathrm{C}} \equiv \partial_{Y} \omega_{X, Y,+}\left(\Phi_{\mathrm{C}}\right)\right|_{X=Y=0} .
\end{aligned}
$$

In words, even if $\mathrm{A}$ and $\mathrm{B}$ are at the same temperature, the temperature differential between $\mathrm{B}$ and $\mathrm{C}$ may cause a heat flux out of A equal to $X L_{\mathrm{A}}+o(X)$ for $X$ small. $L_{\mathrm{C}}$ has the same interpretation. If the GKF in the form (1.1) holds for $\mathrm{L}=\mathrm{A}+\mathrm{B}, \mathrm{R}=\mathrm{C}$ and $A=\Phi_{\mathrm{A}}$, then

$$
L_{\mathrm{A}}=\frac{1}{2} \int_{-\infty}^{\infty} \omega_{\beta}\left(\Phi_{A}\left(\Phi_{\mathrm{C}}\right)_{t}\right) \mathrm{d} t .
$$

Similarly, if the GKF holds for $\mathrm{L}=\mathrm{B}+\mathrm{C}, \mathrm{R}=\mathrm{A}$ and $A=\Phi_{\mathrm{C}}$, then

$$
L_{\mathrm{C}}=\frac{1}{2} \int_{-\infty}^{\infty} \omega_{\beta}\left(\Phi_{\mathrm{C}}\left(\Phi_{A}\right)_{t}\right) \mathrm{d} t=\frac{1}{2} \int_{-\infty}^{\infty} \omega_{\beta}\left(\left(\Phi_{\mathrm{C}}\right)_{t} \Phi_{A}\right) \mathrm{d} t .
$$

Hence, the GKF and the relation

$$
\int_{-\infty}^{\infty} \omega_{\beta}\left(\left[\left(\Phi_{\mathrm{C}}\right)_{t}, \Phi_{\mathrm{A}}\right]\right) \mathrm{d} t=0
$$

which is a well-known consequence of the KMS condition, yield the Onsager reciprocity relations

$$
L_{\mathrm{A}}=L_{\mathrm{C}}
$$

In this paper we give a rigorous axiomatic proof of the GKF (1.1) and the ORR (1.2) in the abstract setting of algebraic quantum statistical mechanics.

The main idea of our proof can be illustrated by the following simple computation. Assume that $\mathrm{L}$ and $\mathrm{R}$ are finite dimensional systems, i.e., that they are described by finite dimensional Hilbert spaces $\mathcal{H}_{\mathrm{L}}, \mathcal{H}_{\mathrm{R}}$ and Hamiltonians $H_{\mathrm{L}}, H_{\mathrm{R}}$. The Hilbert space of the joint system is $\mathcal{H}=\mathcal{H}_{\mathrm{L}} \otimes \mathcal{H}_{\mathrm{R}}$. Let $V$ be a self-adjoint operator 


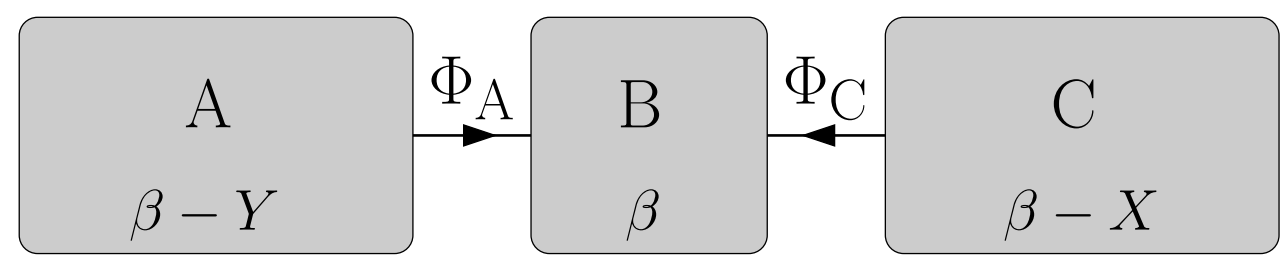

Figure 2: The joint system A $+B+C$.

on $\mathcal{H}$ describing the interaction of $\mathrm{L}$ and $\mathrm{R}$. The Hamiltonian of the joint system is $H=H_{\mathrm{L}}+H_{\mathrm{R}}+V$ and $A_{t}=\mathrm{e}^{\mathrm{i} t H} A \mathrm{e}^{-\mathrm{i} t H}$. The heat flux observable is

$$
\Phi=-\left.\frac{\mathrm{d}}{\mathrm{d} t} \mathrm{e}^{\mathrm{i} t H} H_{\mathrm{R}} \mathrm{e}^{-\mathrm{i} t H}\right|_{t=0}=\mathrm{i}\left[H_{\mathrm{R}}, V\right] .
$$

A common choice for the reference (initial) state of the joint system is the product state $\omega_{\text {ref }}$ with density matrix

$$
\frac{1}{Z} \mathrm{e}^{-\beta H_{\mathrm{L}}-(\beta-X) H_{\mathrm{R}}},
$$

where $Z$ is a normalization constant. As we shall see, in the study of linear response theory a more natural choice is the state $\omega_{X}$ described by the density matrix

$$
\frac{1}{Z} \mathrm{e}^{-\beta H+X H_{\mathrm{R}}}
$$

Let $A$ be an operator on $\mathcal{H}$ and $t>0$. Note that

$$
\omega_{X}\left(A_{t}\right)=\omega_{X}\left(\mathrm{e}^{-\mathrm{i} t\left(H-X H_{\mathrm{R}} / \beta\right)} \mathrm{e}^{\mathrm{i} t H} A \mathrm{e}^{-\mathrm{i} t H} \mathrm{e}^{\mathrm{i} t\left(H-X H_{\mathrm{R}} / \beta\right)}\right),
$$

and so

$$
\omega_{X}\left(A_{t}\right)-\omega_{X}(A)=\frac{X}{\beta} \int_{0}^{t} \omega_{X}\left(\mathrm{i}\left[H_{\mathrm{R}}, A_{s}\right]\right) \mathrm{d} s .
$$

If the system is time-reversal invariant and $A$ is odd under the time-reversal operation, then $\omega_{X}(A)=0$ for all $X$ (and in particular, $\omega_{0}\left(A_{t}\right)=\omega_{0}(A)=0$ for all $t$ ). Hence, (1.4) yields

$$
\left.\partial_{X} \omega_{X}\left(A_{t}\right)\right|_{X=0}=\frac{1}{\beta} \int_{0}^{t} \omega_{\beta}\left(\mathrm{i}\left[H_{\mathrm{R}}, A_{s}\right]\right) \mathrm{d} s .
$$

Another elementary computation yields

$$
\begin{aligned}
\omega_{\beta}\left(\mathrm{i}\left[H_{\mathrm{R}}, A_{s}\right]\right) & =\frac{\mathrm{i}}{Z} \operatorname{Tr}\left(A_{s}\left[e^{-\beta H}, H_{\mathrm{R}}\right]\right)=\frac{\mathrm{i}}{Z} \operatorname{Tr}\left(A_{s}\left[\mathrm{e}^{-\beta H} H_{\mathrm{R}} \mathrm{e}^{\beta H}-H_{\mathrm{R}}\right] \mathrm{e}^{-\beta H}\right) \\
& =\int_{0}^{\beta} \omega_{\beta}\left(A_{s} \mathrm{e}^{-u H} \Phi \mathrm{e}^{u H}\right) \mathrm{d} u,
\end{aligned}
$$

and so

$$
\left.\partial_{X} \omega_{X}\left(A_{t}\right)\right|_{X=0}=\frac{1}{\beta} \int_{0}^{t} \mathrm{~d} s \int_{0}^{\beta} \mathrm{d} u \omega_{\beta}\left(A_{s} \Phi_{\mathrm{i} u}\right) .
$$

Needless to say, only infinitely extended systems have non-trivial thermodynamics. The central point of our argument is that the relation (1.5) can be proven even if the systems $\mathrm{L}$ and $\mathrm{R}$ are infinitely extended and Hamiltonians are not defined any more. We shall show that the relation (1.5) is a general consequence of the KMS-condition 
and holds in the abstract setting of algebraic quantum statistical mechanics under very mild technical conditions. Our argument then continues as follows. Assume that there exists a state $\omega_{X,+}$ on $\mathcal{O}$ such that for any observable A,

$$
\lim _{t \rightarrow+\infty} \omega_{X}\left(A_{t}\right)=\omega_{X,+}(A) .
$$

The state $\omega_{X,+}$ is the non-equilibrium steady state (NESS) of the system $\mathrm{L}+\mathrm{R}$ and is the central object of nonequilibrium statistical mechanics. Let $A$ be an observable for which (1.5) holds. Assume in addition that the function

$$
X \mapsto \omega_{X,+}(A),
$$

is differentiable at $X=0$ and that the limit and the derivative in the expression

$$
\left.\lim _{t \rightarrow+\infty} \partial_{X} \omega_{X}\left(A_{t}\right)\right|_{X=0}
$$

can be interchanged. Then,

$$
\left.\partial_{X} \omega_{X,+}(A)\right|_{X=0}=\frac{1}{\beta} \int_{0}^{\infty} \mathrm{d} t \int_{0}^{\beta} \mathrm{d} u \omega_{\beta}\left(A_{t} \Phi_{\mathrm{i} u}\right)
$$

and this relation is the Green-Kubo formula.

If in addition the joint thermal equilibrium state $\omega_{\beta}$ is mixing, namely if for all $A, B \in \mathcal{O}$,

$$
\lim _{|t| \rightarrow \infty} \omega_{\beta}\left(\tau^{t}(A) B\right)=\omega_{\beta}(A) \omega_{\beta}(B)
$$

then it is not difficult to show that the KMS condition and the time-reversal invariance imply that (1.9) is equivalent to $(1.1)$.

The above derivation requires a comment. The initial state commonly used in the literature is the decoupled product state $\omega_{\text {ref }}$. In this paper we prove the GKF for the NESS $\omega_{X,+}$ associated to the initial state $\omega_{X}$. On physical grounds, one expects that under normal conditions all states normal w.r.t. $\omega_{X}$ evolve to $\omega_{X,+}$ as $t \rightarrow+\infty$ (see [JP3, AJPP1]). Since in particular $\omega_{X}$ and $\omega_{\text {ref }}$ are mutually normal they are physically equivalent initial states.

The three key assumptions of our derivation are the existence of the NESS (1.6), the differentiability of the function (1.7), and the interchange of the limit and the derivative in (1.8). Verification of each of them in physically interesting models is a difficult task.

In the literature two distinct techniques have been used so far in the study of NESS. The first is the scattering approach, initiated by Robinson [Ro1], and further developed in [BM, AM, Ru1, FMU]. The second is the spectral approach developed in [JP2]. In the continuation of this paper [JOP2, JOPP] we will show how the spectral and the scattering approach can be used to verify our abstract assumptions and we will illustrate the general theory on well-known examples of open quantum systems with free fermionic reservoirs studied in [Da, LeSp, BM, AM, JP2, FMU].

Acknowledgment. The research of the first author was partly supported by NSERC. Part of this work was done during the visit of the first and the third author to ESI in Vienna. V.J. and C.-A.P. are grateful to J. Dereziński, G.-M. Graf and J. Yngvason for invitation to the workshop "Open Quantum Systems" and for their hospitality. A part of this work has been done during the visit of the first author to CPT-CNRS. Y.O. is supported by the Japan Society for the Promotion of Science. This work has been done during the stay of Y.O. to CPT-CNRS, partly supported by the Canon Foundation in Europe and JSPS. 


\section{The model and the results}

\subsection{Basic concepts}

In this subsection we briefly review for notational purposes a few basic notions of algebraic quantum statistical mechanics. This topic is discussed in many places in the literature and we will freely use the results described in the classical references [BR1, BR2]. An exposition of the algebraic formalism suited to non-equilibrium quantum statistical mechanics can be found in the reviews [Ru3, JP3, AJPP1].

A $C^{*}$-dynamical system is a pair $(\mathcal{O}, \tau)$, where $\mathcal{O}$ is a $C^{*}$-algebra with identity $\mathbb{1}$ and $\tau^{t}, t \in \mathbb{R}$, is a strongly continuous group of $*$-automorphisms of $\mathcal{O}$. In the context of quantum statistical mechanics, the elements of $\mathcal{O}$ describe observables of a physical system and the group $\tau$ describes their time evolution in the Heisenberg picture. The physical states are described by the states on $\mathcal{O}$, i.e., positive normalized linear functionals in $\mathcal{O}^{*}$. A quantum dynamical system is a triple $(\mathcal{O}, \tau, \omega)$, where $\omega$ is a given reference state. The physical states of thermal equilibrium at inverse temperature $\beta$ are described by $(\tau, \beta)$-KMS states in $\mathcal{O}^{*}$. We shall assume that $\beta>0$ although all our results also apply to $\beta<0$. We recall that $\omega$ is a $(\tau, \beta)$-KMS state if for all $A, B \in \mathcal{O}$ there exists a function $F_{A, B}(z)$, analytic in the strip $0<\operatorname{Im} z<\beta$, bounded and continuous on its closure, and satisfying the KMS-boundary condition

$$
F_{A, B}(t)=\omega\left(A \tau^{t}(B)\right), \quad F_{A, B}(t+\mathrm{i} \beta)=\omega\left(\tau^{t}(B) A\right) .
$$

The three-line theorem yields that

$$
\left|F_{A, B}(z)\right| \leq\|A\|\|B\|
$$

for $0 \leq \operatorname{Im} z \leq \beta$. For notational convenience we shall write $\omega\left(A \tau^{z}(B)\right) \equiv F_{A, B}(z)$.

An anti-linear involutive $*$-automorphism $\Theta: \mathcal{O} \rightarrow \mathcal{O}$ is called time-reversal of $(\mathcal{O}, \tau)$ if

$$
\Theta \circ \tau^{t}=\tau^{-t} \circ \Theta
$$

for all $t \in \mathbb{R}$. A state $\omega$ is called time-reversal invariant if $\omega(\Theta(A))=\omega\left(A^{*}\right)$ for all $A \in \mathcal{O}$.

\subsection{The setup}

We shall consider two quantum dynamical systems $\left(\mathcal{O}_{\mathrm{L}}, \tau_{\mathrm{L}}, \omega_{\mathrm{L}}\right)$ and $\left(\mathcal{O}_{\mathrm{R}}, \tau_{\mathrm{R}}, \omega_{\mathrm{R}}\right)$, which we shall call the left, L, and the right, $\mathrm{R}$, system. We denote the generators of $\tau_{\mathrm{L}}$ and $\tau_{\mathrm{R}}$ by $\delta_{\mathrm{L}}$ and $\delta_{\mathrm{R}}$, i.e., $\tau_{\mathrm{L}}^{t}=\mathrm{e}^{t \delta_{\mathrm{L}}}$ and $\tau_{\mathrm{R}}^{t}=\mathrm{e}^{t \delta_{\mathrm{R}}}$.

If the system $\mathrm{L}$ and $\mathrm{R}$ are brought into contact, then the algebra of observables of the joint system is $\mathcal{O}=$ $\mathcal{O}_{\mathrm{L}} \otimes \mathcal{O}_{\mathrm{R}}$ (our results do not depend on the choice of the cross-norm defining this tensor product). Its decoupled dynamics $\tau_{0}=\tau_{\mathrm{L}} \otimes \tau_{\mathrm{R}}$ is generated by $\delta^{(0)}=\delta_{\mathrm{L}}+\delta_{\mathrm{R}}$.

Notation. If $\mathcal{L}$ is an operator on $\mathcal{O}_{\mathrm{L}}$, then we will denote by the same letter the operator $\mathcal{L} \otimes I$ on $\mathcal{O}_{\mathrm{L}} \otimes \mathcal{O}_{\mathrm{R}}$. Hence, we write $\delta_{\mathrm{L}}$ for $\delta_{\mathrm{L}} \otimes I$, etc. We will use the same convention in the case of $\mathcal{O}_{\mathrm{R}}$.

Let $V \in \mathcal{O}$ be a self-adjoint perturbation describing the interaction of $\mathrm{L}$ and $\mathrm{R}$. The $C^{*}$-dynamical system describing the interacting system $\mathrm{L}+\mathrm{R}$ is $(\mathcal{O}, \tau)$, where the interacting dynamics $\tau$ is generated by

$$
\delta=\delta^{(0)}+\mathrm{i}[V, \cdot] .
$$

In what follows $\beta>0$ is a fixed reference inverse temperature and $\epsilon>0$ is a small number such that $\beta>\epsilon$. We set $I_{\epsilon}=(-\epsilon, \epsilon)$. We make the following assumptions concerning the reference states of $\mathrm{L}$ and $\mathrm{R}$ and the interaction $V$.

(A1) $\omega_{\mathrm{L}}$ is the unique $\left(\tau_{\mathrm{L}}, \beta\right)$-KMS state on $\mathcal{O}_{\mathrm{L}}$. The possible reference states of $\mathrm{R}$ are parametrized by $X \in I_{\epsilon}$ and $\omega_{\mathrm{R}, X}$ is the unique $\left(\tau_{\mathrm{R}}, \beta-X\right)$-KMS state on $\mathcal{O}_{\mathrm{R}}$. We shall write $\omega_{\mathrm{R}, 0}=\omega_{\mathrm{R}}$. 
(A2) $V \in \operatorname{Dom}\left(\delta_{\mathrm{R}}\right)$.

If (A2) holds, then the observable describing the heat flux out of $\mathrm{R}$ is (recall (1.3))

$$
\Phi \equiv \delta_{\mathrm{R}}(V)
$$

Consider the family of states

$$
\omega_{X}^{(0)}=\omega_{\mathrm{L}} \otimes \omega_{\mathrm{R}, X}
$$

Let $\sigma_{X}^{(0)}$ and $\sigma_{X}$ be the $C^{*}$-dynamics on $\mathcal{O}$ generated by

$$
\begin{gathered}
\delta_{X}^{(0)}=\delta^{(0)}-\frac{X}{\beta} \delta_{\mathrm{R}}, \\
\delta_{X}=\delta_{X}^{(0)}+\mathrm{i}[V, \cdot] .
\end{gathered}
$$

$\omega_{X}^{(0)}$ is the unique $\left(\sigma_{X}^{(0)}, \beta\right)$-KMS state on $\mathcal{O}$. The Araki perturbation theory implies that there exists a unique $\left(\sigma_{X}, \beta\right)$-KMS state $\omega_{X}$ on $\mathcal{O}$. The states $\omega_{X}$ and $\omega_{X}^{(0)}$ are mutually normal. Note that $\sigma_{0}=\tau$ and that $\omega_{0}$ is the unique $(\tau, \beta)$-KMS state on $\mathcal{O}$. We denote $\omega_{\beta} \equiv \omega_{0}$.

Our next assumption deals with time-reversal invariance.

(A3) There exists a time-reversal $\Theta$ of $\left(\mathcal{O}, \tau_{0}\right)$ such that

$$
\Theta \circ \tau_{\mathrm{L}}^{t}=\tau_{\mathrm{L}}^{-t} \circ \Theta, \quad \Theta \circ \tau_{\mathrm{R}}^{t}=\tau_{\mathrm{R}}^{-t} \circ \Theta, \quad \Theta(V)=V .
$$

One easily shows that $\Theta$ is also a time-reversal of $(\mathcal{O}, \tau),\left(\mathcal{O}, \sigma_{X}^{(0)}\right)$ and $\left(\mathcal{O}, \sigma_{X}\right)$. If in addition (A1) holds, then the states $\omega_{X}^{(0)}$ and $\omega_{X}$ are time-reversal invariant.

In essence, our main result is:

Theorem 2.1 Suppose that Assumptions (A1)-(A3) hold. Let $A \in \operatorname{Dom}\left(\delta_{\mathrm{R}}\right)$ be a self-adjoint observable such that $\Theta(A)=-A$. Then, for all $t \in \mathbb{R}$, the function

$$
X \mapsto \omega_{X}\left(\tau^{t}(A)\right),
$$

is differentiable at $X=0$, and

$$
\left.\partial_{X} \omega_{X}\left(\tau^{t}(A)\right)\right|_{X=0}=\frac{1}{\beta} \int_{0}^{t} \mathrm{~d} s \int_{0}^{\beta} \mathrm{d} u \omega_{\beta}\left(\tau^{s}(A) \tau^{\mathrm{i} u}(\Phi)\right) .
$$

Remark 1. Assume in addition that the quantum dynamical systems $\left(\mathcal{O}_{\mathrm{R}}, \tau_{\mathrm{R}}, \omega_{\mathrm{R}, X}\right)$ are ergodic for $|X|<\epsilon$. Then, $\left\{\omega_{X}\right\}$ is a family of mutually singular states (see [JP3, AJPP1]) and the differentiability of the function $X \mapsto \omega_{X}\left(\tau^{t}(A)\right)$ at $X=0$ is far from obvious. The somewhat surprising generality of Theorem 2.1 critically depends on the time-reversal assumption which ensures that $\omega_{X}(A)=0$ for all $|X|<\epsilon$. For a related technical point in classical non-equilibrium statistical mechanics we refer the reader to [Ru5].

Remark 2. Let $\omega_{\beta, \lambda}$ denote the $\beta$-KMS state for the $C^{*}$-dynamics generated by $\delta-\lambda\left[\Xi_{t}, \cdot\right]$ where

$$
\Xi_{t} \equiv \int_{0}^{t} \tau^{-s}(\Phi) \mathrm{d} s
$$

Then, by Araki's perturbation theory one has

$$
\left.\frac{1}{\beta} \partial_{\lambda} \omega_{\beta, \lambda}(A)\right|_{\lambda=0}=\left.\partial_{X} \omega_{X}\left(\tau^{t}(A)\right)\right|_{X=0} .
$$

Hence, the finite time $t$ linear response to the thermodynamical force $X$ is equal, up to a factor of $\beta$, to the equilibrium linear response to the mechanical perturbation $\Xi_{t}$. For additional information concerning this point we refer the reader to $[\mathrm{Zu}, \mathrm{TM}]$. 


\subsection{The Green-Kubo formula}

Our next assumption postulates the existence of a NESS w.r.t. the initial state $\omega_{X}$.

(A4) For each $X \in I_{\epsilon}$ there exists a state $\omega_{X,+}$ such that for all $A \in \mathcal{O}$,

$$
\lim _{t \rightarrow+\infty} \omega_{X}\left(\tau^{t}(A)\right)=\omega_{X,+}(A) .
$$

Note that $\omega_{0,+}=\omega_{\beta}$ is the unique $(\tau, \beta)$-KMS state on $\mathcal{O}$.

Finally, we shall assume that our coupled system is mixing at equilibrium.

(A5) For all $A, B \in \mathcal{O}$,

$$
\lim _{|t| \rightarrow \infty} \omega_{\beta}\left(\tau^{t}(A) B\right)=\omega_{\beta}(A) \omega_{\beta}(B)
$$

The observables for which we will establish the Green-Kubo formula are characterized by

Definition 2.2 Assume that (A1) and (A4) hold. Let $A \in \mathcal{O}$ be an observable such that for all $t$ the function

$$
X \mapsto \omega_{X}\left(\tau^{t}(A)\right),
$$

is differentiable at $X=0$. We call such an observable regular if the function

$$
X \mapsto \omega_{X,+}(A),
$$

is differentiable at $X=0$ and

$$
\left.\lim _{t \rightarrow+\infty} \partial_{X} \omega_{X}\left(\tau^{t}(A)\right)\right|_{X=0}=\left.\partial_{X} \omega_{X,+}(A)\right|_{X=0} .
$$

A consequence of Theorem 2.1 and the definition of regular observable is the Green-Kubo formula.

Theorem 2.3 Suppose that Assumptions (A1)-(A4) hold. Let $A \in \operatorname{Dom}\left(\delta_{\mathrm{R}}\right)$ be a regular self-adjoint observable such that $\Theta(A)=-A$. Then

$$
\left.\partial_{X} \omega_{X,+}(A)\right|_{X=0}=\frac{1}{\beta} \int_{0}^{\infty} \mathrm{d} t \int_{0}^{\beta} \mathrm{d} u \omega_{\beta}\left(\tau^{t}(A) \tau^{\mathrm{i} u}(\Phi)\right) .
$$

If in addition (A5) holds, then

$$
\left.\partial_{X} \omega_{X,+}(A)\right|_{X=0}=\frac{1}{2} \int_{-\infty}^{\infty} \mathrm{d} t \omega_{\beta}\left(A \tau^{t}(\Phi)\right)
$$

Remark 1. In (2.15), $\int_{-\infty}^{\infty}=\lim _{T \rightarrow \infty} \int_{-T}^{T}$.

Remark 2. It follows from our proof that linear response to the thermodynamical force $X$ can be computed without time-reversal assumption. Assume that (A1), (A2), (A4) hold and that $A \in \operatorname{Dom}\left(\delta_{\mathrm{R}}\right)$ is a regular observable. Then

$$
\left.\partial_{X} \omega_{X,+}(A)\right|_{X=0}=\left.\partial_{X} \omega_{X}(A)\right|_{X=0}+\frac{1}{\beta} \int_{0}^{\infty} \mathrm{d} t \int_{0}^{\beta} \mathrm{d} u \omega_{\beta}\left(\tau^{t}(A) \tau^{\mathrm{i} u}(\Phi)\right) .
$$

This formula will be discussed in more detail in [JOP1]. 
In applications to concrete models the key assumptions to check are (A4), (A5) and that physically relevant observables are regular. The assumptions (A4) and (A5) have been verified for open quantum systems with free Fermi gas reservoirs in [BM, AM, JP2, FMU]. In these works (A4) is established in the stronger form, i.e. for any $\eta$ normal w.r.t. $\omega_{X}^{(0)}$ and all $A \in \mathcal{O}, \lim _{t \rightarrow+\infty} \eta\left(\tau^{t}(A)\right)=\omega_{X,+}(A)$. In the continuation of this paper [JOP2, JOPP] we will prove that in these models the observables describing heat fluxes within $\mathrm{L}$ are regular.

A simple class of models to which Theorem 2.3 directly applies are quasi-free open systems studied in [AH, AJPP1, AJPP2, AP]. These models are also exactly solvable and the GKF and the ORR can be checked by direct computation.

\subsection{Onsager reciprocity relations}

Consider three $C^{*}$-dynamical systems $\left(\mathcal{O}_{J}, \tau_{J}\right), J=\mathrm{A}, \mathrm{B}, \mathrm{C}$. The generator of $\tau_{J}$ is denoted by $\delta_{J}$. We set

$$
\mathcal{O}=\bigotimes_{J} \mathcal{O}_{J}, \quad \tau_{0}=\bigotimes_{J} \tau_{J}, \quad \delta^{(0)}=\sum_{J} \delta_{J}
$$

Let $V \in \mathcal{O}$ be a self-adjoint perturbation describing the interaction of the joint system and let $\tau$ be the dynamics generated by $\delta=\delta^{(0)}+\mathrm{i}[V, \cdot]$. We shall assume that $V$ has the form

$$
V=V_{\mathrm{AB}}+V_{\mathrm{BC}}
$$

where $V_{\mathrm{AB}} \in \mathcal{O}_{\mathrm{A}} \otimes \mathcal{O}_{\mathrm{B}}$ and $V_{\mathrm{BC}} \in \mathcal{O}_{\mathrm{B}} \otimes \mathcal{O}_{\mathrm{C}}$. If $V_{\mathrm{AB}} \in \operatorname{Dom}\left(\delta_{\mathrm{A}}\right)$ and $V_{\mathrm{BC}} \in \operatorname{Dom}\left(\delta_{\mathrm{C}}\right)$, then the observables describing the heat flow out of $A$ and $C$ are

$$
\Phi_{\mathrm{A}}=\delta_{\mathrm{A}}\left(V_{\mathrm{AB}}\right), \quad \Phi_{\mathrm{C}}=\delta_{\mathrm{C}}\left(V_{\mathrm{BC}}\right),
$$

see Figure 2. Note that in this case $\delta_{\mathrm{C}}\left(\Phi_{\mathrm{A}}\right)=\delta_{\mathrm{A}}\left(\Phi_{\mathrm{C}}\right)=0$.

We assume that (A3) holds in the following form: there exists a time-reversal $\Theta$ of $\left(\mathcal{O}, \tau_{0}\right)$ such that $\tau_{J}^{t} \circ \Theta=$ $\Theta \circ \tau_{J}^{-t}$ for all $J$ and $\Theta\left(V_{\mathrm{AB}}\right)=V_{\mathrm{AB}}, \Theta\left(V_{\mathrm{BC}}\right)=V_{\mathrm{BC}}$. Then $\Theta$ is a time-reversal of the coupled system $(\mathcal{O}, \tau)$ and $\Theta\left(\Phi_{\mathrm{A}}\right)=-\Phi_{\mathrm{A}}, \Theta\left(\Phi_{\mathrm{C}}\right)=-\Phi_{\mathrm{C}}$.

We shall also consider the joint systems $\mathrm{A}+\mathrm{B}$ and $\mathrm{B}+\mathrm{C}$. In the first case the algebra of observables is $\mathcal{O}_{\mathrm{A}} \otimes \mathcal{O}_{\mathrm{B}}$ and the dynamics is generated by $\delta_{\mathrm{A}}+\delta_{\mathrm{B}}+\mathrm{i}\left[V_{\mathrm{AB}}, \cdot\right]$. The system $\mathrm{B}+\mathrm{C}$ is defined in a similar way. Let $\beta>0$ be the fixed inverse temperature.

Consider $\mathrm{L}=\mathrm{A}+\mathrm{B}$ as the left system and $\mathrm{R}=\mathrm{C}$ as the right system. Suppose that Assumptions (A1), (A2), (A4), (A5) hold (we have already assumed the time-reversal invariance) and that the observable $\Phi_{\mathrm{A}}$ is regular. Let $\omega_{\mathrm{A}+\mathrm{B}, \mathrm{C}, X,+}$ be the NESS in the Assumption (A4). Then the kinetic transport coefficient

$$
L_{\mathrm{A}}=\left.\partial_{X} \omega_{\mathrm{A}+\mathrm{B}, \mathrm{C}, X,+}\left(\Phi_{\mathrm{A}}\right)\right|_{X=0},
$$

is well-defined and the Green-Kubo formula holds,

$$
L_{\mathrm{A}}=\frac{1}{2} \int_{-\infty}^{\infty} \omega_{\beta}\left(\Phi_{\mathrm{A}} \tau^{t}\left(\Phi_{\mathrm{C}}\right)\right) \mathrm{d} t,
$$

where $\omega_{\beta}$ is the $(\tau, \beta)$-KMS state on $\mathcal{O}$.

Consider now $\mathrm{L}=\mathrm{B}+\mathrm{C}$ as the left system and $\mathrm{R}=\mathrm{A}$ as the right system. Suppose that Assumptions (A1), (A2), (A4) and (A5) hold and that the observable $\Phi_{\mathrm{C}}$ is regular. Let $\omega_{\mathrm{B}+\mathrm{C}, \mathrm{A}, X,+}$ be the NESS in the Assumption (A4). Then the kinetic transport coefficient

$$
L_{\mathrm{C}}=\left.\partial_{X} \omega_{\mathrm{B}+\mathrm{C}, X,+}\left(\Phi_{\mathrm{C}}\right)\right|_{X=0},
$$

is well-defined and the Green-Kubo formula holds,

$$
L_{\mathrm{C}}=\frac{1}{2} \int_{-\infty}^{\infty} \omega_{\beta}\left(\Phi_{\mathrm{C}} \tau^{t}\left(\Phi_{\mathrm{A}}\right)\right) \mathrm{d} t=\frac{1}{2} \int_{-\infty}^{\infty} \omega_{\beta}\left(\tau^{t}\left(\Phi_{\mathrm{C}}\right) \Phi_{\mathrm{A}}\right) \mathrm{d} t .
$$


Since $\omega_{\beta}$ is mixing, by the well-known stability criterion (Theorem 5.4.12 in [BR2]),

$$
\lim _{T \rightarrow \infty} \int_{-T}^{T} \omega_{\beta}\left(\left[\tau^{t}\left(\Phi_{\mathrm{C}}\right), \Phi_{\mathrm{A}}\right]\right) \mathrm{d} t=0
$$

and so

$$
L_{\mathrm{A}}=L_{\mathrm{C}}
$$

This is the Onsager reciprocity relations for heat fluxes.

\section{Proofs}

Throughout this subsection we assume that (A1) and (A2) hold.

We start with:

Lemma 3.1 Assume that (A3) holds. Then:

(1) $\Theta$ is a time-reversal of the $C^{*}$-dynamical systems $(\mathcal{O}, \tau)$ and $\left(\mathcal{O}, \sigma_{X}\right)$,

(2) The states $\omega_{X}$ are time-reversal invariant.

(3) $\Theta(\Phi)=-\Phi$.

Proof. (1) Recall that

$$
\tau^{t}(A)=\Gamma_{t} \tau_{0}^{t}(A) \Gamma_{t}^{*},
$$

where $\Gamma_{t} \in \mathcal{O}$ is the family of unitary elements defined by

$$
\Gamma_{t}=\mathbb{1}+\sum_{n \geq 1}(\mathrm{i} t)^{n} \int_{0 \leq s_{n} \leq \cdots \leq s_{1} \leq 1} \tau_{0}^{t s_{n}}(V) \cdots \tau_{0}^{t s_{1}}(V) \mathrm{d} s_{1} \cdots \mathrm{d} s_{n},
$$

see Proposition 5.4.1. in [BR2]. Similarly,

$$
\tau^{-t}(A)=\hat{\Gamma}_{t} \tau_{0}^{-t}(A) \hat{\Gamma}_{t}^{*}
$$

where

$$
\hat{\Gamma}_{t}=\mathbb{1}+\sum_{n \geq 1}(-\mathrm{i} t)^{n} \int_{0 \leq s_{n} \leq \cdots \leq s_{1} \leq 1} \tau_{0}^{-t s_{n}}(V) \cdots \tau_{0}^{-t s_{1}}(V) \mathrm{d} s_{1} \cdots \mathrm{d} s_{n} .
$$

Since $\Theta(V)=V$ and $\Theta\left(\Gamma_{t}\right)=\hat{\Gamma}_{t}$, we have that $\Theta \circ \tau^{t}=\tau^{-t} \circ \Theta$, i.e., $\Theta$ is a time-reversal of $(\mathcal{O}, \tau)$.

Since $\Theta$ is also a time-reversal of $\left(\mathcal{O}, \sigma_{X}^{(0)}\right)$, one proves in the same way that $\Theta$ is a time-reversal of $\left(\mathcal{O}, \sigma_{X}\right)$.

(2) Set

$$
\tilde{\omega}_{X}(A)=\omega_{X}\left(\Theta\left(A^{*}\right)\right) .
$$

One easily checks that $\tilde{\omega}_{X}$ is a $\left(\sigma_{X}, \beta\right)$-KMS state. Assumption (A1) and Araki's perturbation theory imply that $\omega_{X}$ is the unique $\left(\sigma_{X}, \beta\right)$-KMS state on $\mathcal{O}$. Hence, $\tilde{\omega}_{X}=\omega_{X}$.

(3) is an immediate consequence of the relation $\Theta\left(\tau_{\mathrm{R}}^{t}(V)\right)=\tau_{\mathrm{R}}^{-t}(V)$ and the definition of $\Phi$.

Lemma 3.2 The group $\tau$ preserves $\operatorname{Dom}\left(\delta_{\mathrm{R}}\right)$ and for $A \in \operatorname{Dom}\left(\delta_{\mathrm{R}}\right)$ the function

$$
\mathbb{R} \ni t \mapsto \delta_{\mathrm{R}}\left(\tau^{t}(A)\right),
$$

is norm continuous. 
Proof. Since $V \in \operatorname{Dom}\left(\delta_{\mathrm{R}}\right)$, the formula (3.17) yields that $\Gamma_{t} \in \operatorname{Dom}\left(\delta_{\mathrm{R}}\right)$ and that

$$
\delta_{\mathrm{R}}\left(\Gamma_{t}\right)=\sum_{n \geq 1}(\mathrm{i} t)^{n} \int_{0 \leq s_{n} \leq \cdots \leq s_{1} \leq 1} \sum_{j} \tau_{0}^{t s_{n}}(V) \cdots \tau_{0}^{t s_{j}}\left(\delta_{\mathrm{R}}(V)\right) \cdots \tau_{0}^{t s_{1}}(V) \mathrm{d} s_{1} \cdots \mathrm{d} s_{n} .
$$

The series on the right is uniformly convergent for $t$ in compact sets, and so $\mathbb{R} \ni t \mapsto \delta_{\mathrm{R}}\left(\Gamma_{t}\right)$ is a norm continuous function. The formula (3.16) yields

$$
\delta_{\mathrm{R}}\left(\tau^{t}(A)\right)=\delta_{\mathrm{R}}\left(\Gamma_{t}\right) \tau_{0}^{t}(A) \Gamma_{t}^{*}+\Gamma_{t} \tau_{0}^{t}\left(\delta_{\mathrm{R}}(A)\right) \Gamma_{t}^{*}+\Gamma_{t} \tau_{0}^{t}(A) \delta_{\mathrm{R}}\left(\Gamma_{t}\right)^{*}
$$

and the statement follows.

In the remaining part of the proof we will need to approximate $A$ and $V$ by analytic elements. For this reason we briefly recall a few basic facts about such analytic approximations (see [Ar, BR2, DJP]). For $C \in \mathcal{O}$ we set

$$
C_{j}=\sqrt{\frac{j}{\pi}} \int_{\mathbb{R}} \mathrm{e}^{-j t^{2}} \tau_{0}^{t}(C) \mathrm{d} t, \quad j=1,2, \cdots
$$

Every $C_{j}$ is an analytic element for the group $\tau_{0}$, i.e., the function $t \mapsto \tau_{0}^{t}\left(C_{j}\right) \in \mathcal{O}$ extends to an entire analytic function on $\mathbb{C}$. Moreover,

$$
\left\|C_{j}\right\| \leq\|C\| \quad \text { and } \quad \lim _{j \rightarrow \infty}\left\|C_{j}-C\right\|=0 .
$$

If in addition $C \in \operatorname{Dom}\left(\delta_{\mathrm{R}}\right)$, then

$$
\delta_{\mathrm{R}}\left(C_{j}\right)=\sqrt{\frac{j}{\pi}} \int_{\mathbb{R}} \mathrm{e}^{-j t^{2}} \tau_{0}^{t}\left(\delta_{\mathrm{R}}(C)\right) \mathrm{d} t, \quad j=1,2, \cdots
$$

and so $\delta_{\mathrm{R}}\left(C_{j}\right)$ is also an analytic element for $\tau_{0}^{t}$ and $\lim _{j \rightarrow \infty}\left\|\delta_{\mathrm{R}}\left(C_{j}\right)-\delta_{\mathrm{R}}(C)\right\|=0$.

Lemma 3.3 For $A \in \operatorname{Dom}\left(\delta_{\mathrm{R}}\right)$,

$$
\sigma_{X}^{t}(A)-\tau^{t}(A)=-\frac{X}{\beta} \int_{0}^{t} \sigma_{X}^{t-s}\left(\delta_{\mathrm{R}}\left(\tau^{s}(A)\right)\right) \mathrm{d} s .
$$

Proof. Assume first that $A \in \operatorname{Dom}(\delta) \cap \operatorname{Dom}\left(\delta_{\mathrm{R}}\right)$. Then,

$$
\frac{\mathrm{d}}{\mathrm{d} t} \sigma_{X}^{-t}\left(\tau^{t}(A)\right)=\sigma_{X}^{-t}\left(\left(\delta-\delta_{X}\right)\left(\tau^{t}(A)\right)\right)=\frac{X}{\beta} \sigma_{X}^{-t}\left(\delta_{\mathrm{R}}\left(\tau^{t}(A)\right)\right)
$$

and (3.20) holds.

Let now $A \in \operatorname{Dom}\left(\delta_{\mathrm{R}}\right)$ and let $A_{j}$ be given by (3.18). Clearly, $A_{j} \in \operatorname{Dom}(\delta) \cap \operatorname{Dom}\left(\delta_{R}\right)$ and (3.20) holds for $A_{j}$. The relations

$$
\begin{aligned}
\delta_{\mathrm{R}}\left(\tau^{t}\left(A_{j}\right)\right) & =\delta_{\mathrm{R}}\left(\Gamma_{t} \tau_{0}^{t}\left(A_{j}\right) \Gamma_{t}^{*}\right) \\
& =\delta_{\mathrm{R}}\left(\Gamma_{t}\right) \tau_{0}^{t}\left(A_{j}\right) \Gamma_{t}^{*}+\Gamma_{t} \tau_{0}^{t}\left(\delta_{\mathrm{R}}\left(A_{j}\right)\right) \Gamma_{t}^{*}+\Gamma_{t} \tau_{0}^{t}\left(A_{j}\right) \delta_{\mathrm{R}}\left(\Gamma_{t}\right)^{*},
\end{aligned}
$$

yield that $\lim _{j \rightarrow \infty} \delta_{\mathrm{R}}\left(\tau^{t}\left(A_{j}\right)\right)=\delta_{\mathrm{R}}\left(\tau^{t}(A)\right)$ uniformly for $t$ in compact sets, and the statement follows.

Since $\operatorname{Dom}\left(\delta_{\mathrm{R}}\right)$ is dense in $\mathcal{O}$, Lemma 3.3 yields that for all $A \in \mathcal{O}$,

$$
\lim _{X \rightarrow 0}\left\|\sigma_{X}^{t}(A)-\tau^{t}(A)\right\|=0 .
$$


Lemma 3.4 For all $A \in \mathcal{O}$,

$$
\lim _{X \rightarrow 0} \omega_{X}(A)=\omega_{\beta}(A)
$$

Proof. Let $\mathcal{L}$ be the set of weak* limit points of the net $\left\{\omega_{X}\right\}$ as $X \rightarrow 0$. The set $\mathcal{L}$ is non-empty since the unit ball in $\mathcal{O}^{*}$ is weak* compact. Relation (3.22) and Proposition 5.3.25 in [BR2] yield that every element of $\mathcal{L}$ is a $(\tau, \beta)$-KMS state. Assumption (A1) implies that $\omega_{\beta}$ is the unique $(\tau, \beta)$-KMS state on $\mathcal{O}$ and so $\mathcal{L}=\left\{\omega_{\beta}\right\}$.

Lemma 3.5 Assume that (A3) holds. Let $A \in \operatorname{Dom}\left(\delta_{\mathrm{R}}\right)$ be a self-adjoint observable such that $\Theta(A)=-A$. Then for all $t \in \mathbb{R}$ the function

$$
X \mapsto \omega_{X}\left(\tau^{t}(A)\right)
$$

is differentiable at $X=0$ and

$$
\left.\partial_{X} \omega_{X}\left(\tau^{t}(A)\right)\right|_{X=0}=\frac{1}{\beta} \int_{0}^{t} \omega_{\beta}\left(\delta_{\mathrm{R}}\left(\tau^{s}(A)\right)\right) \mathrm{d} s .
$$

Proof. Since $\omega_{X}$ is $\sigma_{X}$-invariant, Lemma 3.3 yields

$$
\frac{\omega_{X}\left(\tau^{t}(A)\right)-\omega_{X}(A)}{X}=\frac{1}{\beta} \int_{0}^{t} \omega_{X}\left(\delta_{\mathrm{R}}\left(\tau^{s}(A)\right)\right) \mathrm{d} s .
$$

By Lemma 3.1, $\omega_{X}(A)=\omega_{X}(\Theta(A))=-\omega_{X}(A)$, and so $\omega_{X}(A)=0$. Similarly, since $\omega_{0}=\omega_{\beta}$ is $\tau$-invariant, $\omega_{0}\left(\tau^{t}(A)\right)=\omega_{0}(A)=0$ for all $t$. Hence,

$$
\frac{\omega_{X}\left(\tau^{t}(A)\right)-\omega_{0}\left(\tau^{t}(A)\right)}{X}=\frac{1}{\beta} \int_{0}^{t} \omega_{X}\left(\delta_{\mathrm{R}}\left(\tau^{s}(A)\right)\right) \mathrm{d} s .
$$

This relation, Lemmas 3.2, 3.4, and the dominated convergence yield the statement.

Lemma 3.6 Assume that $A \in \operatorname{Dom}\left(\delta_{\mathrm{R}}\right)$. Then

$$
\omega_{\beta}\left(\delta_{\mathrm{R}}(A)\right)=\int_{0}^{\beta} \omega_{\beta}\left(A \tau^{\mathrm{i} s}(\Phi)\right) \mathrm{d} s .
$$

Proof. We will freely use standard results of Araki's theory of perturbation of KMS-states (see [Ar, BR2, DJP]). Let $V_{j}$ be the analytic approximations of $V$ given by (3.18). Since $V \in \operatorname{Dom}\left(\delta_{\mathrm{R}}\right), \Phi_{j} \equiv \delta_{\mathrm{R}}\left(V_{j}\right)$ are analytic approximations of the heat flux observable $\Phi=\delta_{\mathrm{R}}(V)$. Note also that the function $t \mapsto \Gamma_{t, j}$, where

$$
\Gamma_{t, j}=\mathbb{1}+\sum_{n \geq 1}(\mathrm{i} t)^{n} \int_{0 \leq s_{n} \leq \cdots \leq s_{1} \leq 1} \tau_{0}^{t s_{n}}\left(V_{j}\right) \cdots \tau_{0}^{t s_{1}}\left(V_{j}\right) \mathrm{d} s_{1} \cdots \mathrm{d} s_{n},
$$

extends to an entire analytic $\mathcal{O}$-valued function

$$
\Gamma_{z, j}=\mathbb{1}+\sum_{n \geq 1}(\mathrm{i} z)^{n} \int_{0 \leq s_{n} \leq \cdots \leq s_{1} \leq 1} \tau_{0}^{z s_{n}}\left(V_{j}\right) \cdots \tau_{0}^{z s_{1}}\left(V_{j}\right) \mathrm{d} s_{1} \cdots \mathrm{d} s_{n} .
$$

The function $z \mapsto \Gamma_{z, j}^{*}$ is also entire analytic and for all $z, \Gamma_{z, j} \Gamma_{z, j}^{*}=\mathbb{1}$. 
Let $\tau_{j}$ be the $C^{*}$-dynamics on $\mathcal{O}$ generated by $\delta^{(0)}+\mathrm{i}\left[V_{j}, \cdot\right]$, and let $\omega_{j}$ be the unique $\left(\tau_{j}, \beta\right)$-KMS state. Since for any $C \in \mathcal{O}$,

$$
\tau_{j}^{t}(C)=\Gamma_{t, j} \tau_{0}^{t}(C) \Gamma_{t, j}^{*},
$$

we conclude that $A_{j}, V_{j}$, and $\Phi_{j}$ are also analytic elements for $\tau_{j}$ and that $\lim _{j \rightarrow \infty}\left\|\tau_{j}^{t}-\tau\right\|=0$.

We shall prove first that for all $j$,

$$
\omega_{j}\left(\delta_{\mathrm{R}}\left(A_{j}\right)\right)=\int_{0}^{\beta} \omega_{j}\left(A_{j} \tau_{j}^{\mathrm{i} u}\left(\Phi_{j}\right)\right) \mathrm{d} u .
$$

This formula combined with a limiting argument $j \rightarrow \infty$ will yield the statement.

The following relations hold

$$
\begin{aligned}
\frac{\mathrm{d}}{\mathrm{d} z} \Gamma_{z, j} & =\mathrm{i} \Gamma_{z, j} \tau_{0}^{z}\left(V_{j}\right), \\
\frac{\mathrm{d}}{\mathrm{d} z} \Gamma_{z, j}^{*} & =-\mathrm{i} \tau_{0}^{z}\left(V_{j}\right) \Gamma_{z, j}^{*} .
\end{aligned}
$$

One easily verifies that for all $z \in \mathbb{C}, \Gamma_{z, j} \in \operatorname{Dom}\left(\delta_{\mathrm{R}}\right), \Gamma_{\bar{z}, j}^{*} \in \operatorname{Dom}\left(\delta_{\mathrm{R}}\right)$, that the functions $z \mapsto \delta_{\mathrm{R}}\left(\Gamma_{z, j}\right)$, $z \mapsto \delta_{\mathrm{R}}\left(\Gamma_{\bar{z}, j}^{*}\right)$ are entire analytic, and that

$$
\begin{aligned}
& \frac{\mathrm{d}}{\mathrm{d} z} \delta_{\mathrm{R}}\left(\Gamma_{z, j}\right)=\mathrm{i} \delta_{\mathrm{R}}\left(\Gamma_{z, j}\right) \tau_{0}^{z}\left(V_{j}\right)+\mathrm{i} \Gamma_{z, j} \tau_{0}^{z}\left(\Phi_{j}\right), \\
& \frac{\mathrm{d}}{\mathrm{d} z} \delta_{\mathrm{R}}\left(\Gamma_{\bar{z}, j}^{*}\right)=-\mathrm{i} \tau_{0}^{z}\left(\Phi_{j}\right) \Gamma_{z, j}^{*}-\mathrm{i} \tau_{0}^{z}\left(V_{j}\right) \delta_{\mathrm{R}}\left(\Gamma_{\bar{z}, j}^{*}\right) .
\end{aligned}
$$

Relations (3.25) and (3.26) yield

$$
\frac{\mathrm{d}}{\mathrm{d} z} \Gamma_{z, j} \delta_{\mathrm{R}}\left(\Gamma_{\bar{z}, j}^{*}\right)=-\mathrm{i} \Gamma_{z, j} \tau_{0}^{z}\left(\Phi_{j}\right) \Gamma_{\bar{z}, j}^{*}=-\mathrm{i} \tau_{j}^{z}\left(\Phi_{j}\right)
$$

Recall that $\omega_{0}^{(0)}=\omega_{\mathrm{L}} \otimes \omega_{\mathrm{R}}$. By the basic identity of Araki's perturbation theory, for any $C \in \mathcal{O}$,

$$
\omega_{j}(C)=\frac{\omega_{0}^{(0)}\left(C \Gamma_{\mathrm{i} \beta, j}\right)}{\omega_{0}^{(0)}\left(\Gamma_{\mathrm{i} \beta, j}\right)} .
$$

In particular,

$$
\omega_{j}\left(\delta_{\mathrm{R}}\left(A_{j}\right)\right)=\frac{\omega_{0}^{(0)}\left(\delta_{\mathrm{R}}\left(A_{j}\right) \Gamma_{\mathrm{i} \beta, j}\right)}{\omega_{0}^{(0)}\left(\Gamma_{\mathrm{i} \beta, j}\right)} .
$$

Since $\omega_{0}^{(0)}\left(\delta_{\mathrm{R}}(C)\right)=0$ for any $C \in \operatorname{Dom}\left(\delta_{\mathrm{R}}\right)$, we have

$$
\omega_{0}^{(0)}\left(\delta_{\mathrm{R}}\left(A_{j}\right) \Gamma_{\mathrm{i} \beta, j}\right)=-\omega_{0}^{(0)}\left(A_{j} \delta_{\mathrm{R}}\left(\Gamma_{\mathrm{i} \beta, j}\right)\right)=-\omega_{0}^{(0)}\left(A_{j}\left[\delta_{\mathrm{R}}\left(\Gamma_{\mathrm{i} \beta, j}\right) \Gamma_{-\mathrm{i} \beta, j}^{*}\right] \Gamma_{\mathrm{i} \beta, j}\right) .
$$

Hence,

$$
\omega_{j}\left(\delta_{\mathrm{R}}\left(A_{j}\right)\right)=\omega_{j}\left(A_{j}\left[\Gamma_{\mathrm{i} \beta, j} \delta_{\mathrm{R}}\left(\Gamma_{-\mathrm{i} \beta, j}^{*}\right)\right]\right) .
$$

Relation (3.27) implies

$$
\frac{\mathrm{d}}{\mathrm{d} u} \Gamma_{\mathrm{i} u, j} \delta_{\mathrm{R}}\left(\Gamma_{-\mathrm{i} u, j}^{*}\right)=\tau_{j}^{\mathrm{i} u}\left(\Phi_{j}\right),
$$


and so

$$
\Gamma_{\mathrm{i} \beta, j} \delta_{\mathrm{R}}\left(\Gamma_{-\mathrm{i} \beta, j}^{*}\right)=\int_{0}^{\beta} \tau_{j}^{\mathrm{i} u}\left(\Phi_{j}\right) \mathrm{d} u .
$$

Relations (3.28) and (3.29) yield (3.24).

It remains to show that

$$
\begin{aligned}
\lim _{j \rightarrow \infty} \omega_{j}\left(\delta_{\mathrm{R}}\left(A_{j}\right)\right) & =\omega_{\beta}\left(\delta_{\mathrm{R}}(A)\right), \\
\lim _{j \rightarrow \infty} \int_{0}^{\beta} \omega_{j}\left(A_{j} \tau_{j}^{\mathrm{i} u}\left(\Phi_{j}\right)\right) \mathrm{d} u & =\int_{0}^{\beta} \omega_{\beta}\left(A \tau^{\mathrm{i} u}(\Phi)\right) \mathrm{d} u .
\end{aligned}
$$

By the properties of analytic approximations,

$$
\lim _{j \rightarrow \infty}\left\|\omega_{j}-\omega_{\beta}\right\|=0 \quad \text { and } \quad \lim _{j \rightarrow \infty}\left\|\delta_{\mathrm{R}}\left(A_{j}\right)-\delta_{\mathrm{R}}(A)\right\|=0,
$$

and these relations yield (3.30). To prove (3.31) we argue as follows.

For $t \in \mathbb{R}$ we set

$$
h_{j}(t)=\int_{0}^{\beta} \omega_{j}\left(A_{j} \tau_{j}^{\mathrm{i} u+t}\left(\Phi_{j}\right)\right) \mathrm{d} u, \quad h(t)=\int_{0}^{\beta} \omega_{\beta}\left(A \tau^{\mathrm{i} u+t}(\Phi)\right) \mathrm{d} u .
$$

Then

$$
\begin{aligned}
\int_{0}^{t} \omega_{j}\left(\left[A_{j}, \tau_{j}^{u}\left(\Phi_{j}\right)\right]\right) \mathrm{d} u & =\int_{0}^{t} \omega_{j}\left(A_{j} \tau_{j}^{u}\left(\Phi_{j}\right)\right) \mathrm{d} u-\int_{0}^{t} \omega_{j}\left(A_{j} \tau_{j}^{u+\mathrm{i} \beta}\left(\Phi_{j}\right)\right) \mathrm{d} u \\
& =\mathrm{i} \int_{0}^{\beta} \omega_{j}\left(A_{j} \tau_{j}^{\mathrm{i} u}\left(\Phi_{j}\right)\right) \mathrm{d} u-\mathrm{i} \int_{0}^{\beta} \omega_{j}\left(A_{j} \tau_{j}^{\mathrm{i} u+t}\left(\Phi_{j}\right)\right) \mathrm{d} u \\
& =\mathrm{i}\left(h_{j}(0)-h_{j}(t)\right) .
\end{aligned}
$$

In the first step we have used the KMS condition and in the second that the integral of the function $z \mapsto$ $\omega_{j}\left(A_{j} \tau_{j}^{z}\left(\Phi_{j}\right)\right)$ over the boundary of the rectangle with vertices $0, t, t+\mathrm{i} \beta, \mathrm{i} \beta$ is zero. Similarly,

$$
\int_{0}^{t} \omega_{\beta}\left(\left[A, \tau^{u}(\Phi)\right]\right) \mathrm{d} u=\mathrm{i}(h(0)-h(t)) .
$$

Since

$$
\lim _{j \rightarrow \infty} \omega_{j}\left(\left[A_{j}, \tau_{j}^{u}\left(\Phi_{j}\right)\right]\right)=\omega_{\beta}\left(\left[A, \tau^{u}(\Phi)\right]\right)
$$

we derive that for all $t \in \mathbb{R}$

$$
\lim _{j \rightarrow \infty}\left(h_{j}(t)-h_{j}(0)\right)=h(t)-h(0) .
$$

Note that

$$
\begin{aligned}
\int_{-\infty}^{\infty} \mathrm{e}^{-t^{2}} h_{j}(t) \mathrm{d} t & =\int_{0}^{\beta} \mathrm{d} u \int_{-\infty}^{\infty} \mathrm{d} t \mathrm{e}^{-(t-\mathrm{i} u)^{2}} \omega_{j}\left(A_{j} \tau_{j}^{t}\left(\Phi_{j}\right)\right), \\
\int_{-\infty}^{\infty} \mathrm{e}^{-t^{2}} h(t) \mathrm{d} t & =\int_{0}^{\beta} \mathrm{d} u \int_{-\infty}^{\infty} \mathrm{d} t \mathrm{e}^{-(t-\mathrm{i} u)^{2}} \omega_{\beta}\left(A \tau^{t}(\Phi)\right)
\end{aligned}
$$

Since for all $t$,

$$
\lim _{j \rightarrow \infty} \omega_{j}\left(A \tau_{j}^{t}\left(\Phi_{j}\right)\right)=\omega_{\beta}\left(A \tau^{t}(\Phi)\right),
$$


we conclude that

$$
\lim _{j \rightarrow \infty} \int_{-\infty}^{\infty} \mathrm{e}^{-t^{2}}\left(h_{j}(t)-h(t)\right) \mathrm{d} t=0
$$

Then (3.32) and (3.33) yield

$$
\lim _{j \rightarrow \infty} h_{j}(0)=h(0)
$$

and (3.31) follows.

Proof of Theorem 2.1. This theorem is an immediate consequence of Lemmas 3.5 and 3.6.

Proof of Theorem 2.3. Relation (2.14) follows from Theorem 2.1 and the definition of regular observable.

To prove (2.15), we need to show that

$$
\lim _{t \rightarrow+\infty} \frac{1}{\beta} \int_{0}^{\beta}\left[\int_{0}^{t} \omega_{\beta}\left(\tau^{s}(A) \tau^{\mathrm{i} u}(\Phi)\right) \mathrm{d} s\right] \mathrm{d} u=\frac{1}{2} \int_{-\infty}^{\infty} \omega_{\beta}\left(A \tau^{t}(\Phi)\right) \mathrm{d} t .
$$

Time-reversal invariance yields that for $s, u \in \mathbb{R}$,

$$
\omega_{\beta}\left(\tau^{s}(A) \tau^{u}(\Phi)\right)=\omega_{\beta}\left(\tau^{s}(\Theta(A)) \tau^{u}(\Theta(\Phi))\right)=\omega_{\beta}\left(\Theta\left(\tau^{-s}(A) \tau^{-u}(\Phi)\right)\right)=\omega_{\beta}\left(\tau^{-u}(\Phi) \tau^{-s}(A)\right) .
$$

This identity and the KMS-condition imply

$$
\omega_{\beta}\left(\tau^{s}(A) \tau^{u}(\Phi)\right)=\omega_{\beta}\left(\tau^{-s}(A) \tau^{\mathrm{i} \beta-u}(\Phi)\right) .
$$

The analytic continuation yields that for $s \in \mathbb{R}$ and $u \in[0, \beta]$,

$$
\omega_{\beta}\left(\tau^{s}(A) \tau^{\mathrm{i} u}(\Phi)\right)=\omega_{\beta}\left(\tau^{-s}(A) \tau^{\mathrm{i} \beta-\mathrm{i} u}(\Phi)\right),
$$

and so

$$
\begin{aligned}
\frac{1}{\beta} \int_{0}^{\beta}\left[\int_{0}^{t} \omega_{\beta}\left(\tau^{s}(A) \tau^{\mathrm{i} u}(\Phi)\right) \mathrm{d} s\right] \mathrm{d} u & =\frac{1}{2 \beta} \int_{0}^{\beta}\left[\int_{-t}^{t} \omega_{\beta}\left(\tau^{s}(A) \tau^{\mathrm{i} u}(\Phi)\right) \mathrm{d} s\right] \mathrm{d} u \\
& =\frac{1}{2 \beta} \int_{0}^{\beta}\left[\int_{-t}^{t} \omega_{\beta}\left(A \tau^{s+\mathrm{i} u}(\Phi)\right) \mathrm{d} s\right] \mathrm{d} u
\end{aligned}
$$

The integral of the function

$$
z \mapsto \omega_{\beta}\left(A \tau^{z}(B)\right)
$$

over the boundary of the rectangle with vertices $-t, t, t+\mathrm{i} u,-t+\mathrm{i} u$ is zero. Hence,

$$
\int_{-t}^{t} \omega_{\beta}\left(A \tau^{s+\mathrm{i} u}(\Phi)\right) \mathrm{d} s=\int_{-t}^{t} \omega_{\beta}\left(A \tau^{s}(\Phi)\right) \mathrm{d} s+R(t, u)
$$

where

$$
R(t, u)=\mathrm{i} \int_{0}^{u}\left[\omega_{\beta}\left(A \tau^{t+\mathrm{i} y}(\Phi)\right)-\omega_{\beta}\left(A \tau^{-t+\mathrm{i} y}(\Phi)\right)\right] \mathrm{d} y,
$$

and we derive

$$
\frac{1}{\beta} \int_{0}^{\beta}\left[\int_{0}^{t} \omega_{\beta}\left(\tau^{s}(A) \tau^{\mathrm{i} u}(\Phi)\right) \mathrm{d} s\right] \mathrm{d} u=\frac{1}{2} \int_{-t}^{t} \omega_{\beta}\left(A \tau^{s}(\Phi)\right) \mathrm{d} s+\frac{1}{2 \beta} \int_{0}^{\beta} R(t, u) \mathrm{d} u .
$$


Assumption (A5) implies that

$$
\lim _{t \rightarrow+\infty} \omega_{\beta}\left(A \tau^{ \pm t+\mathrm{i} y}(\Phi)\right)=\omega_{\beta}(A) \omega_{\beta}(\Phi)=0
$$

This fact and the dominated convergence theorem yield

$$
\lim _{t \rightarrow+\infty} \sup _{0 \leq u \leq \beta}|R(t, u)|=0
$$

and Relation (3.34) implies the statement.

\section{References}

[Ar] Araki, H.: Relative Hamiltonians for faithful normal states of a von Neumann algebra. Publ. R.I.M.S., Kyoto Univ. 9, 165 (1973).

[AH] Araki, H., Ho, T.G: Asymptotic time evolution of a partitioned infinite two-sided isotropic $X Y$-chain. Tr. Mat. Inst. Steklova, 228 Probl. Sovrem. Mat. Fiz., 203, (2000); translation in Proc. Steklov Inst. Math. 228, 191, (2000).

[AP] Aschbacher, W., Pillet, C-A.: Non-equilibrium steady states of the $X Y$ chain. J. Stat. Phys. 12, 1153 (2003).

[AJPP1] Aschbacher, W., Jakšić, V., Pautrat, Y., Pillet, C.-A.: Topics in non-equilibrium quantum statistical mechanics. To appear in Lecture Notes in Mathematics.

[AJPP2] Aschbacher, W., Jakšić, V., Pautrat, Y., Pillet, C.-A.: Transport properties of ideal Fermi gases (in preparation).

[AM] Aizenstadt, V.V., Malyshev, V.A.: Spin interaction with an ideal Fermi gas. J. Stat. Phys. 48, 51 (1987).

[BM] Botvich, D.D., Malyshev, V.A.: Unitary equivalence of temperature dynamics for ideal and locally perturbed Fermi gas. Commun. Math. Phys. 61, 209 (1978).

[BGKS] Bouclet, J.M., Germinet, F., Klein, A., and Schenker, J.H.: Linear response theory for magnetic Schrödinger operators in disordered media. J. Funct. Anal. (in press).

[Br] Brenig, W.: Statistical Theory of Heat. Springer-Verlag Berlin (1989).

[BR1] Bratteli, O., Robinson, D. W.: Operator Algebras and Quantum Statistical Mechanics 1. Springer-Verlag, Berlin (1987).

[BR2] Bratteli, O., Robinson, D. W.: Operator Algebras and Quantum Statistical Mechanics 2. Second edition, SpringerVerlag, Berlin (1996).

[Da] Davies, E.B.: Markovian master equations. Commun. Math. Phys. 39, 91 (1974).

[DGM] De Groot, S.R., Mazur, P.: Non-Equilibrium Thermodynamics. North-Holland, Amsterdam (1969).

[DJP] Dereziński, J., Jakšić, V., Pillet, C.-A.: Perturbation theory of $W^{*}$-dynamics, KMS-states and Liouvilleans. Rev. Math. Phys. 15, 447 (2003).

[FMU] Fröhlich, J., Merkli, M., Ueltschi, D.: Dissipative transport: thermal contacts and tunneling junctions. Ann. Henri Poincaré 4, 897 (2004).

[GVV1] Goderis, D., Verbeure, A., Vets, P.: About the exactness of the linear response theory. Commun. Math. Phys. 136, 265 (1991).

[GVV2] Goderis, D., Verbeure, A., Vets, P.: Theory of quantum fluctuations and the Onsager relations. J. Stat. Phys. 56, 721 (1989).

[JOP1] Jakšić, V., Ogata, Y., Pillet, C.-A.: The linear response theory in quantum statistical mechanics. In preparation.

[JOP2] Jakšić, V., Ogata, Y., Pillet, C.-A.: In preparation.

[JOPP] Jakšić, V., Ogata, Y., Pautrat, Y., Pillet, C.-A.: In preparation.

[JP1] Jakšić, V., Pillet, C-A.: On entropy production in quantum statistical mechanics. Commun. Math. Phys. 217, 285 (2001). 
[JP2] Jakšić, V., Pillet, C.-A.: Non-equilibrium steady states for finite quantum systems coupled to thermal reservoirs. Commun. Math. Phys. 226, 131 (2002).

[JP3] Jakšić, V., Pillet, C.-A.: Mathematical theory of non-equilibrium quantum statistical mechanics. J. Stat. Phys. 108, 787 (2002).

[KTH] Kubo, R., Toda, M., Hashitsune, N.: Statistical Physics II. Second edition, Springer-Verlag, Berlin (1991).

[LeSp] Lebowitz, J., Spohn, H.: Irreversible thermodynamics for quantum systems weakly coupled to thermal reservoirs. Adv. Chem. Phys. 39, 109 (1978).

[RB] Rey-Bellet, L.: Open Classical Systems. To appear in Lecture Notes in Mathematics.

[Ro1] Robinson, D.W.: Return to equilibrium. Commun. Math. Phys. 31, 171 (1973).

[Ru1] Ruelle, D.: Natural nonequilibrium states in quantum statistical mechanics. J. Stat. Phys. 98, 57 (2000).

[Ru2] Ruelle, D.: Entropy production in quantum spin systems. Commun. Math. Phys. 224, 3 (2001).

[Ru3] Ruelle, D.: Topics in quantum statistical mechanics and operator algebras. Preprint, mp-arc 01-257 (2001).

[Ru4] Ruelle, D.: Smooth dynamics and new theoretical ideas in nonequilibrium statistical mechanics. J. Stat. Phys. 95, 393 (1999).

[Ru5] Ruelle, D.: Differentiation of SRB states. Commun. Math. Phys. 187, 227 (1997).

[TM] Tasaki, S., Matsui, T.: Fluctuation theorem, nonequilibrium steady states and MacLennan-Zubarev ensembles of a class of large quantum systems. Fundamental Aspects of Quantum Physics (Tokyo, 2001). QP-PQ: Quantum Probab. White Noise Anal., 17, 100. World Sci., River Edge NJ, (2003).

[Zu] Zubarev, D.N.: Nonequilibrium statistical thermodynamics. Consultant Bureau, NY (1974).

[ZMR1] Zubarev, D. N., Morozov, V. G., Röpke, G.: Statistical Mechanics of Nonequilibrium Processes I. Academie Verlag, Berlin (1996).

[ZMR2] Zubarev, D. N., Morozov, V. G., Röpke, G.: Statistical Mechanics of Nonequilibrium Processes II. Academie Verlag, Berlin (1997). 